\title{
REVIEW
}

by Wladyslaw CICHOCKI

University of New Brunswick, Fredericton, New Brunswick, Canada*

cicho@unb.ca

Côté, Marie-Hélène, Remco Knoolhuizen \& John Nerbonne (eds) (2016) The future of dialects. Selected papers from "Methods in Dialectology XV" (Language Variation 1), Berlin: Language Science Press, 411 pp. DOI:10.17169/langsci.b81.78

The International Conference on Methods in Dialectology meets every three years to discuss advances in the study of dialects. The organization of these conferences is "informally" directed by a steering committee that is not aligned with a particular association or learned society. This setting encourages a relaxed and dynamic exchange of research information that comes from a broad range of theoretical and methodological frameworks and that has a large number of international participants. Generally referred to as Methods $X V$, the 15th meeting of this group was held in Groningen, Netherlands, in August 2014, and attracted over 150 oral presentations and posters; 22 of these papers are included in this volume. In keeping with the Methods spirit of collegial exchange, the papers are open-access and can be downloaded at no cost. Also noteworthy is the fact that this volume is the first title to appear in the "Language Variation" series by the Language Science Press, which intends to promote a tradition of open-access publication.

The "future of dialects" theme given in the title of this book is timely; however, only a small number of papers actually address it. Most contributions deal with what one might label as the "future of dialectology;" they present methodological innovations for the analysis of dialect data, especially in the areas of quantification,

\footnotetext{
* Department of French, University of New Brunswick, P.O. Box 4400, Fredericton, NB, CANADA E3B5A3.
} 
mapping and methods of data elicitation. The list of languages analyzed is impressive; it ranges from Breton to Yiddish and includes dialect situations in Europe, North America and Japan. In this review it is not possible to discuss in detail each of the 22 contributions; I will instead highlight five main themes that I identified as I read the papers.

\section{The Future of Dialects}

In the context of forces such as urban marginalization and non-integration, increasing social mobility, and greater access to formal education, traditional dialects are undergoing unexpected changes. Two papers directly address the study of these so-called "future" dialects. The "new" varieties described in both studies have their origins in contact situations, and the authors are searching for appropriate methods to analyze them. Nagy studies heritage languages that have been brought by immigrants to Canada. As these languages develop across several generations, they diverge from the parent varieties and form "new" dialects; examples are "Canadian Chinese," "Italiese" (Canadian Italian) and "Ukish" (Canadian Ukrainian). Nagy proposes to adapt the methods of variationist sociolinguistics to study both the linguistic and attitudinal bases of these "new" varieties. Belgian Dutch tussentaal represents a different kind of "future" dialect. Ghyselen describes this variety in the context of a shift from diglossia to a diaglossic repertoire; that is, speakers who usually choose dialect variants in informal situations and standard variants in formal settings are starting to use forms that are intermediate between standard and dialect; this shift occurs in informal supraregional situations. While all the speakers in Ghyselen's corpus are participating in this shift, very few of them have a strong association to these intermediate diaglossic variants, making it difficult for the researcher to isolate this "new" variety. Ghyselen argues for more observation of speech in informal supraregional speech settings.

Three other papers also contribute to the "future of dialects" theme. Škevin appeals to semiotics to explain the dialect levelling that is taking place in the lexicon of a local variety of Čakavian Croatian. Romance-based lexical variants are losing vitality 
in part because of a change in semiotic space; the concepts that these lexical items signify are losing their place of importance in the daily lives of speakers, which leads to a reduction in the number of users of the variants as well as to cultural and dialect levelling. Ota, Nikaido and Utsugi study a change in the tonal system of Kagoshima Japanese, a regional variety that is being influenced by Standard Japanese. The change in accented and unaccented word patterns is realized by new tones that are intermediate between standard and regional varieties and that reflect both dedialectalization and de-standardization. Hirano describes Japanese-English codeswitching in conversations between native speakers of English who live in Japan. She uses social network analysis to explain how to an increase in the use of these codeswitches is linked to the changing identities of the speakers.

\section{Quantification}

In their analyses of dialect data researchers apply basic descriptive statistics as well as advanced techniques such as transformations, logistic regression and principal components analysis. Researchers are also using quantitative methods to address issues in dialectological inquiry.

One of the important questions in dialectometry - how to identify and rank the linguistic features that define the dialect areas that have been determined by quantitative analysis - is a central theme of several papers. Montemagni and Wieling describe an approach that combines cluster analysis with two metrics that measure the distinctiveness and representativeness of linguistic features. They use these metrics to isolate sets of lexical items that are most "important" in characterizing varieties of Tuscan Italian. Bloem, Wieling and Nerbonne apply the same distinctiveness and representativeness metrics to study pronunciation errors by $L 2$ learners of English. Their application identifies specific pronunciation features that characterize Dutch, French and Hungarian learners; their findings overlap closely with results found in the L2 acquisition literature. Factorial-type multivariate statistical analyses can also be used effectively to identify and rank linguistic features. Pickel 
applies factor analysis to obtain several orthogonal rankings of features that are associated with dialect areas in Bavarian Swabia (in Germany). In her study of Belgian Dutch tussentaal Ghyselen uses multiple correspondence analysis to measure the association of individual speakers to clusters of linguistic features that define this "new" variety. In a related study, Brun-Trigaud, Solliec and Le Dû carry out a number of applications of the Levenshtein algorithm to measure linguistic distances in data found in a linguistic atlas of Breton (spoken in France); their goal is to determine the degree to which linguistic distances are influenced by one high-frequency feature as opposed to several features with lower frequencies of occurrence.

Other researchers apply dialectometric techniques to investigate well-known problems in dialectological analysis. Matthusek shows how fieldworker effects, such as idiosyncrasies in phonetic transcription, "create" isoglosses that can significantly mislead the analysis of dialect structures. Wieling and Montemagni address the question of whether quantitative analysis should include or exclude low frequency forms, which are generally considered to add "noise" to an analysis. They show that including infrequent forms does not alter the configuration of the major dialect regions established by quantitative analysis.

\section{Mapping}

Researchers apply various techniques to map linguistic data onto geographic space. Many of these techniques are available in software packages that are in the public domain such as Gabmap, developed at the University of Groningen (see the papers by Mathussek and by Montemagni and Wieling), and GeoLing, developed at the Universities of Augsburg and UIm (see the paper by Pickl).

Two papers present original mapping tools that are useful for exploratory analysis. Kumagai uses "animation" to play sequences of geographic maps that display degrees of lexical similarity among the 2400 localities in the Linguistic Atlas of Japan Database. This technique allows the researcher to look for explanatory factors; the animation visualizes patterns of transitions in geographic space onto which one can superimpose information about road networks and feudal boundaries. Fukushima also 
uses simultaneous mapping to trace real-time changes. Combining data from four separate surveys that span almost 100 years in the Niigata prefecture of Japan, she illustrates the geographic trajectories of lexical changes that are either complete or in progress.

\section{Phonetics, Syntax and Data Elicitation}

Even though the linguistic atlas continues to be the main source of data in dialectological research, new kinds of data are being studied thanks to innovative technologies and new data elicitation techniques. Especially noteworthy are innovations found in the study of phonetics and syntax.

Perceptual phonetics is a growing area in phonetic science. Kendall and Fridland study the perception of different vowels in US English and find clear regional patterns based on sound perception; however, these patterns do not align in a simple way with established regional patterns based on sound production. A recent innovation in phonetic analysis is the use of ultrasound imaging: ultrasound video at a high frame rate provides images of articulatory movements that can be used to analyze temporal changes and co-articulation effects. Carignan, Mielke and Dodsworth use ultrasound tongue imaging to study patterns of tongue movements in the tensing of North American English /æ/; among their findings are regional differences in the timing of tongue raising before velars. Spreafico applies the same technique to study the tongue shape of Italian /s/ and its variable realizations by Italian-dominant, Tyrolean-dominant and balanced Italian-Tyrolean bilingual speakers.

Syntactic data continue to receive increasing attention from dialectologists. In a novel approach to the study of morphosyntactic features in British English, Wolk and Szmrecsanyi combine the study of pre-determined morphosyntactic variables, as found in different corpora including the linguistic atlas, with a bottom-up approach that examines bigrams of part-of-speech tags, found in corpora of spontaneous speech. Bigrams are the co-occurrences of two-word collocations. The bigram approach is interesting because it can be generalized to an n-gram (i.e., n-word 
collocations) and because the features that are analyzed are not determined in an $a$ priori fashion but emerge from the data observed. The combination of different data sources for the study of syntactic variation is also the theme of Llop Naya's work on negation in Catalan. She combines data from speaker recordings, dialect literature, grammaticality judgements and meta-linguistic comments by native informants. Stoeckle examines 26 morphosyntactic features in the Syntactic Atlas of Germanspeaking Switzerland. He measures the use of the dominant (i.e., most frequent) variant of each of these features across geographic regions and among all speakers within a region in order to establish areas that are "hot spots" of syntactic variation. An interesting result is that regions with high syntactic variation are those that have generally been considered as very conservative.

Imitation and dubbing offer original methods for the elicitation of dialect data. In their study of imitation data, Schäffer, Leser and Cysouw analyze both the target language and the imitator's own language in order to identify which features are salient for listeners who imitate closely related language varieties. Lara Bermejo shows how dubbing can be used to elicit a large number of tokens of variables that might otherwise have very low frequencies of occurrence. He asks speakers to dub scenes shown on videos to elicit ustedes variable in Andalusian Spanish.

\section{Theoretic Issues}

Pickl revisits the definition of "dialect area" employing prototype theory to argue that dialect areas are not crisp but fuzzy. This leads him to suggest that certain quantitative techniques - such as factor analysis, as opposed to cluster analysis - are more appropriate than others for research that aims to identify dialect areas. Onishi discusses two tenets of the wave theory of linguistic change: that change is gradual and continuing, and that it spreads from central to peripheral areas. Studying variables from the Linguistic Atlas of Japan, he is able to illustrate the opposite of both tenets: the spread of a change can occur with a rapid burst, and change can also spread from peripheral to central areas. 


\section{Conclusion}

On the whole, this volume illustrates very effectively that current dialectological inquiry is a dynamic, multidisciplinary enterprise that continues to adapt exciting methodological innovations. The success of the volume in communicating this dynamism is due in no small part to the careful work of the co-editors and of the large group of referees who have ensured the high quality of these papers. All 22 contributions - as well as the editors' detailed overview - have extensive and up-todate bibliographies. The papers are polished and well written; indeed, only a few require some additional attention to the details of English grammar and orthography. Both the general reader and the specialist researcher will find that the research presented in this volume is informative and stimulating. 